\title{
URSS vs. EEUU, RDA vs. RFA: Guerra Fría en los Juegos Olímpicos de Verano (1952-1988) U.S.S.R. vs. USA, GDR vs. FRG: Cold War at the Summer Olympics (1952-1988) \\ *Mateo Rodríguez Quijada, **Svetlana Molkova \\ *Universidade de Santiago de Compostela (España), **Universidad A Coruña (España)
}

\begin{abstract}
Resumen. Durante la Guerra Fría (1947-1991), las cuestiones geopolíticas determinaron el desarrollo del deporte internacional. Los Juegos Olímpicos se convirtieron en la arena de la lucha no solo deportiva sino política. Las victorias deportivas se utilizaban para mostrar la supremacia política, económica e ideológica de los países participantes. En la presente investigación se realiza un análisis de los medallistas de la Unión de Repúblicas Socialistas Soviéticas (URSS), los Estados Unidos (EEUU), la República Democrática Alemana (RDA) y la República Federal de Alemania (RFA) en los Juegos Olímpicos de Verano entre los años 1952 y 1988. Se analiza una muestra de 1945 medallistas olímpicos provenientes de los países indicados que compitieron durante 8 ediciones de los Juegos Olímpicos. Se excluyen del estudio los Juegos de Moscú 1980 y Los Ángeles 1984 debido a los boicots políticos por parte de EEUU y la RFA, y de la URSS y la RDA respectivamente. Los resultados del estudio muestran una clara superioridad de la URSS frente a los EE.UU. en casi todas las características estudiadas. La RDA, a su vez, supera a la RFA en la mayoría de variables analizadas. Los resultados ponen de manifiesto una supremacía de los países del bloque del este en los Juegos Olímpicos de Verano durante la Guerra Fría. Palabras clave: URSS; EEUU; RDA; RFA; Juegos Olímpicos; medallas.
\end{abstract}

\begin{abstract}
During the Cold War (1947-1991), geopolitical issues influenced international sports events. The Olympic Games became a space not only for sports competitions, but also for political clashes. Sports victories were used to pinpoint political, economic and ideological supremacy of the participating countries. The aim of the present research was to analyze the Summer Olympic medalists from the Union of Soviet Socialist Republics (U.S.S.R.), United States of America (USA), the German Democratic Republic (GDR) and the Federal Republic of Germany (FRG) in the period 19521988. We analyzed 1,945 Olympic medalists from above-mentioned countries, competing during 8 Summer Olympic Games. The 1980 Summer Olympics in Moscow and the 1984 Summer Olympics in Los Angeles were excluded due to political boycotts by the USA and the FRG and by the U.S.S.R. and the GDR, respectively. The results of our research show clear superiority of U.S.S.R in comparison to USA in almost all characteristics studied. GDR was better than FRG in most of the analyzed variables. Our outcomes reveal the supremacy of the Eastern Bloc countries at the Summer Olympics during the Cold War.
\end{abstract}

Key words: U.S.S.R; USA; GDR; FRG; Olympic Games; medals.

\section{Introducción}

Desde los inicios de la Guerra Fría, las cuestiones geopolíticas determinaron múltiples facetas del deporte internacional (Soares, 2011). El mundo se dividió en dos bloques: el primero, oriental o comunista, liderado por la Unión de Repúblicas Socialistas Soviéticas, y el segundo, occidental o capitalista, encabezado por los Estados Unidos de América (Swift, 2008). Durante el período de la Guerra Fría (1947-1991), la pertenencia de los países a uno de dichos bloques afectó a todas las esferas de relaciones internacionales, y el ámbito del deporte no fue una excepción(Thomas, 2007).

Ambas potencias competían por la supremacía mundial, y los Juegos Olímpicos presentaban la ocasión ideal para mostrar las fortalezas de cada uno de los sistemas, así como destacar las debilidades de los adversarios (Carreño, 2012). Las competiciones y los éxitos deportivos se convirtieron en instrumentos empleados para propagar sus ideologías políticas (Conlin, 1994; Kioussis \& Hunt, 2015), transformándose, paradójicamente, en una de las pocas salidas culturales que constantemente rompían la Cortina de Hierro (McDougall, 2015). En la lucha entre ambas potencias se utilizaba con frecuencia a la prensa para criticar y ridiculizar los éxitos del rival. La prensa soviética acusaba al deporte occidental de ser elitista, de utilizar métodos tayloristas, y afirmaba que únicamente el modelo socialista encarnaba el verdadero espíritu olímpico, por su parte, las potencias occidentales criticaban la supuesta superioridad soviética, acusando a los entrenadores de someter a los deportistas a controles y prácticas antidemocráticas (Bolz, 2015).

Los niveles de tensión política entre las dos superpotencias alcanzaron sus máximos en los boicots a los Juegos de Moscú 1980 y Los Ángeles 1984. En los primeros, la invasión de Afganistán por parte de las tropas soviéticas en diciembre de 1979 provocó que 67 países (entre ellos, los EE.UU. y la RFA) renunciaran a participar en los Juegos. Como represalia a dicho boicot, la URSS y la gran mayoría de países del este (entre ellos, la RDA) se negaron a acudir a los Juegos de 1984

Fecha recepción: 06-10-16. Fecha de aceptación: 09-03-17

Mateo Rodríguez Quijada

mateo.rodriguez@rai.usc.es

\section{(Laforge, 2012; Meisinger, 2015).}

La competividad y la exigencia de victorias eran tan altas que los valores culturales, sociales y educativos (Robles \& Martínez, 2013) de los Juegos Olímpicos llegaron a desvirtuarse. El objetivo de obtener medallas sustituyó las principales finalidades de los Juegos, que consistían, según Coubertin (1973), en competir lo mejor posible y establecerse como un instrumento pedagógico de primer nivel (Martínez et al., 2016) que permite un desarrollo positivo en los jóvenes (Holt \& Neely, 2011). En múltiples ocasiones, para conseguir las medallas, los deportistas recurrirían al dopaje; por este motivo deporte y dopaje pasaron a ser términos íntimamente relacionados, igual que sucede en la actualidad (Álvarez et al., 2017); sin embargo, mientras que la Unión de Repúblicas Socialistas Soviéticas y la República Democrática Alemana eran más permisivas con dichos métodos -debido a que los deportistas contaban con supervisión médica durante estas prácticas-, los deportistas de los Estados Unidos y de la República Federal de Alemania se veían obligados a acudir al mercado negro -dadas las políticas antidopaje vigentes- con el consiguiente peligro para la salud (Hunt et al., 2014).

El medallero de los Juegos Olímpicos se convirtió en un fiel reflejo del panorama político y económico a nivel mundial: las principales potencias mundiales ocupaban los primeros puestos por recuento de medallas obtenidas. Cada éxito deportivo era utilizado por los gobiernos y por los medios afines para alimentar la lucha de este contra oeste (Morais \& Todd, 2013). Por esta razón la política deportiva de los países de ambos bloques estaba centrada, en gran medida, en la obtención del máximo número de medallistas olímpicos, persiguiendo el objetivo de demostrar su superioridad no solo deportiva, sino también económica, política y logística (Chinchilla, 2009). Considerando, además, que los Juegos Olímpicos son uno de los acontecimientos deportivos más destacados a nivel mundial (Brundage, 1973; Olivera, 2012)sus gestas tienen un gran impacto en la vida de los seres humanos asentándose en su memoria (Bucur et al., 2015) y a los deportistas vencedores se les eleva a la categoría de dioses (Platonov, 2001)-, se puede discernir el motivo por el que la URSS y los EE.UU. luchaban por demostrar su supremacía mundial en dichas competiciones intentando conseguir más medallas que el resto.

\section{Objetivo de la investigación}

A la hora de plantear el presente estudio se decidió optar por el siguiente objetivo de investigación: realizar un análisis de los medallistas 
de la Unión de Repúblicas Socialistas Soviéticas (URSS), los Estados Unidos (EE.UU.), la República Democrática Alemana (RDA) y la República Federal de Alemania (RFA), haciendo comparación en dos bloques -la URSS frente a los EE.UU y la RDA frente a la RFA- en los Juegos Olímpicos de verano (1952-1988).

\section{Material y método}

La muestra del estudio está compuesta de 1945 medallistas olímpicos provenientes de los países de la Unión de Repúblicas Socialistas Soviéticas, los Estados Unidos, la República DemocráticaAlemana y la República Federal deAlemania, que compitieron durante 8 ediciones de los Juegos Olímpicos celebradas entre los años 1952 (primera vez que participa la URSS) y 1988 (última vez que participa la URSS). Cabe señalar que únicamente se analizaron los Juegos Olímpicos de verano y que se excluyeron de este análisis los Juegos de Moscú 1980 y Los Ángeles 1984 debido a los boicots políticos que impidieron, en primer caso, la presencia de los EE.UU. y la RFA (entre otros países), y causaron la ausencia de la URSS y la RDA (entre otros países) en la segunda ocasión. Esta decisión se explica por la ausencia de la competencia directa entre los países implicados en este estudio en las ediciones mencionadas de los Juegos, de allí que los datos no serían relevantes pudiendo contaminar los resultados de la investigación.

Se optó por incluir en esta investigación a los medallistas de la RDA y de la RFA debido a la gran importancia geopolítica e ideológica que tenían estos países como máximos representantes secundarios de ambos bloques y, además, dado que su convivencia y sus relaciones generaron momentos de gran tensión a lo largo de toda la Guerra Fría (Swift, 2008). Otra razón de la inclusión de dichos países en el estudio es la importancia que tuvo el deporte como elemento pacificador para la Alemania de posguerra, permitiendo formar a los grandes medallistas olímpicos durante la Guerra Fría (Bolz, 2015). Como se ha mencionado antes, sin embargo, el análisis de los resultados se realiza entre la URSS y los EE.UU., por un lado, y entre la RDA y la RFA, por otro lado.

Los datos fueron obtenidos del sitio web oficial del Movimiento Olímpico (http://www.olympic.org/). En el análisis de los datos obtenidos se utilizó el software informático de análisis estadístico SPSS versión 22 para Windows. La clasificación de las especialidades deportivas empleada en la presente investigación fue propuesta por Blázquez \& Hernández (1984) que agrupa a los deportes en individuales, de oposición, de cooperación y de cooperación-oposición. La presente clasificación ya ha sido empleada en investigaciones similares, como por ejemplo, la realizada por Rodríguez \& Molkova (2016).

\section{Resultados}

El primer paso del análisis es la obtención de los datos acerca de la participación de la URSS, los EE.UU., la RDAy la RFAen las ediciones mencionadas de los Juegos Olímpicos (tabla 1).

Como se aprecia en la tabla 1, la URSS y los EE.UU. participaron en todos los Juegos Olímpicos entre 1952 y 1988, con la excepción de los Juegos de 1980 -en el caso de la URSS- y los Juegos de 1984 -en el caso de los EE.UU.- por boicots políticos. Por su parte, la RDA y la RFA comenzaron a participar en los Juegos Olímpicos en 1968, en México; anteriormente ambos países estaban integrados bajo la denominación de Equipo Alemán Unificado. La RFA y la RDA tampoco participaron en los Juegos de 1980 y 1984 respectivamente debido a los boicots políticos.

La tabla 2 presenta el número de medallas de cada tipo y la cantidad total de premios conseguidos por los deportistas de cada país. De acuerdo con los datos, la URSS es el país con el mayor número de medallas -815 en su totalidad-, seguida por los EE.UU. con 701, la RDA con 280 y, por último, la RFA con 149 premios. Igualmente, se aprecia que la URSS ha conseguido el mayor número de medallas de oro -310 en total-; en el segundo lugar según el recuento de las medallas de oro se hallan los EE.UU., con 291 premios, y la RDA con 106 medallas de oro superara a la RFA con 39.
Tabla 1.

\begin{tabular}{|c|c|c|c|c|c|}
\hline \multicolumn{2}{|c|}{ Participación en los JJ.OO. } & URSS & EE.UU. & RDA & RFA \\
\hline \multicolumn{2}{|c|}{ Helsinki 1952} & Sí & Sí & No & No \\
\hline \multirow{2}{*}{\multicolumn{2}{|c|}{ Melbourne-Estocolmo 1956}} & Sí & Sí & No & No \\
\hline & & Sí & Sí & No & No \\
\hline \multicolumn{2}{|c|}{ Tokio 1964} & Sí & Sí & No & No \\
\hline \multicolumn{2}{|c|}{ México 1968} & Sí & Sí & Sí & Sí \\
\hline \multicolumn{2}{|c|}{ Múnich 1972} & Sí & Sí & Sí & Sí \\
\hline \multicolumn{2}{|c|}{ Montreal 1976} & Sí & Sí & Sí & Sí \\
\hline \multicolumn{2}{|c|}{ Moscú 1980} & Sí & No & Sí & No \\
\hline \multirow{2}{*}{\multicolumn{2}{|c|}{ Los Ángeles 1984}} & No & Sí & No & Sí \\
\hline & Seúl 1988 & Sí & Sí & Sí & Sí \\
\hline \\
\hline \multicolumn{4}{|c|}{$\begin{array}{l}\text { Tipo de medalla y país. } \\
\text { URSS }\end{array}$} & & RFA \\
\hline Oro & 310 & 291 & 106 & & 39 \\
\hline Plata & 250 & 217 & 92 & & 48 \\
\hline Bronce & 255 & 193 & 82 & & 62 \\
\hline Total & 815 & 701 & 280 & & 149 \\
\hline
\end{tabular}

Tabla 3.

\begin{tabular}{ccccc} 
Número de medallas en las ediciones de los JJ.OO. & & & \\
\hline URSS & EE.UU. & RDA & RFA \\
\hline Helsinki 1952 & 71 & 76 & - & - \\
Melbourne-Estocolmo 1956 & 98 & 72 & - & - \\
Roma 1960 & 103 & 72 & - & - \\
Tokio 1964 & 96 & 91 & - & - \\
México 1968 & 91 & 107 & 25 & 26 \\
Múnich 1972 & 99 & 94 & 65 & 41 \\
Montreal 1976 & 125 & 94 & 90 & 39 \\
Seúl 1988 & 132 & 95 & 100 & 43 \\
\hline
\end{tabular}

Tabla 4.

\begin{tabular}{ccccc}
\multicolumn{5}{c}{ Número de medallas por categorías deportivas. } \\
\hline URSS & EE.UU. & RDA & RFA \\
\hline Masculina & 600 & 541 & 145 & 109 \\
Femenina & 214 & 160 & 135 & 38 \\
Mixta & 1 & 0 & 0 & 2 \\
\hline
\end{tabular}

Tabla 5.

Número de medallas en distintas especialidades deportivas.

\begin{tabular}{|c|c|c|c|c|}
\hline & URSS & EE.UU. & RDA & RFA \\
\hline Atletismo & 152 & 211 & 78 & 33 \\
\hline Ciclismo & 5 & 0 & 2 & 3 \\
\hline Ciclismo en pista & 13 & 1 & 10 & 6 \\
\hline Esgrima & 41 & 1 & 1 & 11 \\
\hline Gimnasia artística & 160 & 2 & 24 & 1 \\
\hline Tiro al blanco & 44 & 23 & 10 & 10 \\
\hline Natación & 37 & 210 & 62 & 11 \\
\hline Tenis & 0 & 5 & 0 & 2 \\
\hline Halterofilia & 54 & 23 & 8 & 4 \\
\hline Lucha Grecorromana & 52 & 1 & 4 & 3 \\
\hline Tiro con arco & 4 & 7 & 0 & 0 \\
\hline Hípica & 7 & 16 & 0 & 21 \\
\hline Fútbol & 4 & 0 & 2 & 1 \\
\hline Remo & 30 & 24 & 34 & 11 \\
\hline Vela & 9 & 25 & 5 & 6 \\
\hline Waterpolo & 6 & 2 & 0 & 0 \\
\hline Baloncesto & 10 & 10 & 0 & 0 \\
\hline Boxeo & 43 & 42 & 7 & 5 \\
\hline Saltos & 8 & 51 & 3 & 0 \\
\hline Lucha libre & 47 & 29 & 2 & 3 \\
\hline Hockey & 0 & 0 & 0 & 2 \\
\hline Pentatlón moderno & 12 & 3 & 0 & 0 \\
\hline Piragüismo & 43 & 6 & 22 & 8 \\
\hline Balonmano & 4 & 0 & 1 & 0 \\
\hline Judo & 18 & 6 & 4 & 8 \\
\hline Voleibol & 10 & 1 & 1 & 0 \\
\hline Gimnasia rítmica & 2 & 0 & 0 & 0 \\
\hline Natación sincronizada & 0 & 2 & 0 & 0 \\
\hline \multicolumn{5}{|c|}{$\begin{array}{l}\text { Tabla } 6 . \\
\text { Número de medallas en distintas modalidades deportivas. }\end{array}$} \\
\hline & URSS & EE.UU. & RDA & RFA \\
\hline Individual & 477 & 495 & 186 & 72 \\
\hline Oposición & 181 & 79 & 18 & 25 \\
\hline Cooperación & 123 & 114 & 72 & 49 \\
\hline Cooperación-oposición & 34 & 13 & 4 & 3 \\
\hline
\end{tabular}

Los datos acerca del número de medallas conseguidas por cada país en distintas ediciones de los JJ.OO se ofrecen en la tabla 3. Se observa que el mayor número de medallas en una edición de los JJ..OO. fue conseguido por la URSS, cuyos deportistas ganaron 132 medallas a lo largo de los Juegos de Seúl, en 1988. La comparación por países revela que, en la cantidad de medallas, la URSS superó a los EE.UU. en 6 de 8 Juegos analizados. La RDA, a su vez, superó a la RFAen 3 de 4 Juegos en los que se enfrentaron.

La tabla 4 permite conocer el número de medallas obtenidas por cada país en diferentes categorías deportivas. Según se aprecia, la URSS es el país con el mayor número de medallistas masculinos (600) y femeninos (214), mientras que la RFAes el país que más medallas ganó en categoría mixta (2) durante los Juegos investigados. Cabe indicar que ni los EEUU ni la RDA consiguieron medallas en categoría mixta.

La tabla 5 presenta datos sobre el número de medallistas de cada país en función de distintas especialidad deportiva. De acuerdo con los datos, la especialidad deportiva en la URSS ganó el mayor número de medallas es gimnasia artística (160), seguida por el atletismo (152) y por 
la halterofilia (54). Por su parte, las especialidades deportivas en las que más medallas consiguieron los deportistas de los EE.UU. son el atletismo (211), la natación (210) y los saltos (51). En cuanto a la RDA, las especialidades con más deportistas galardonados son el atletismo (78), la natación (62) y el remo (34); y, en el caso de la RFA, es el atletismo (33), seguido por la hípica (21) y la esgrima (11), la natación (11) y el remo (11).

Porúltimo, en la tabla 6 se observa el número medallistas en función de la clasificación de la modalidad deportiva propuesta por Blázquez \& Hernández (1984). Como se puede comprobar, la URSS es el país que más medallas tiene en las modalidades deportivas de oposición, cooperación y cooperación-oposición, mientras que los EE.UU. destaca por el mayor número de medallistas en la modalidad individual. Si se compara la RDA con la RFA, se aprecia que la RDA consiguió más medallas en la modalidad individual, la modalidad de cooperación y la de cooperación-oposición, mientras que en la modalidad de oposición son más numerosos los medallistas de la RFA.

\section{Discusión y conclusiones}

La presente investigación analiza a los medallistas olímpicos de la Unión de Repúblicas Socialistas Soviéticas, los Estados Unidos de América, la República Democrática Alemana y la República Federal Alemana durante los Juegos Olímpicos de Verano en los que dichos países participaron. El análisis de los resultados se realiza entre la URSS y los EE.UU., por un lado, y entre la RDA y la RFA, por otro lado.

Los resultados de la investigación señalan que, en cuanto a la comparación entre la URSS y los EE.UU., se aprecia una clara superioridad deportiva de la URSS frente a los EE.UU. en los Juegos Olímpicos de Verano. La URSS obtuvo un total de 815 medallas en los 8 Juegos en los que participó frente a los 701 que consiguieron los EE.UU., y la URSS superó a su rival en 6 de los 8 Juegos en los que se enfrentaron. En relación con el tipo de medalla, la potencia comunista superó a la capitalista en el número de medallas tanto de oro, como de plata y de bronce. En lo referente a la categoría deportiva (masculina, femenina y mixta), la URSS, igualmente, consiguió más medallas en todas las categorías. Por último, en cuanto a la clasificación de la modalidad deportiva, la URSS supera a los EE.UU. en la modalidad de oposición, la de cooperación y la de cooperación-oposición; mientras que en el número de medallistas en la categoría deportiva individual los EE.UU. superaron a la URSS.

En cuanto a la comparación entre la RDA y la RFA, se aprecia un caso similar al anterior, en el que la potencia oriental es superior a la occidental. La RDA consiguió un total de 280 medallas frente a las 149 de la RFA, superándola en 3 de los 4 Juegos en los que se enfrentaron. Atendiendo al tipo de medallas conseguidas, la RDAsuperó a la RFAen la cantidad de medallas de oro, plata y bronce; cabe mencionar, además, que es llamativa la diferencia entre el número de medallas de oro conseguidas por ambos países (106 de la RDA frente a 39 de la RFA). Con respecto a la categoría deportiva, la RDA supera a la RFAen las categorías masculina y femenina, mientras que la RFA lidera la categoría mixta; también es considerable la diferencia entre la cantidad de medallas conseguidas por ambos países en categoría femenina (135 de la RDA frente a 38 de la RFA). Por último, en lo referente a la clasificación de las modalidades deportivas, la RDA obtuvo más medallas en la modalidad individual, la de cooperación y la de cooperación-oposición, mientras que, en la modalidad de oposición, son los deportistas de la RFA quienes ganaron más medallas.

Los resultados de la presente investigación indican la superioridad deportiva de los países comunistas (URSS y RDA) en los Juegos Olímpicos de Verano durante la Guerra Fría, confirmando lo expuesto por Sores (2011), Carreño (2012), Laforge (2012), Morais \& Todd (2013) y Pieper (2014). El mayor número de medallas conseguidas por la URSS y la distribución más equilibrada de sus deportistas entre diversas especialidades y modalidades deportivas puede atribuirse principalmente al plan de nacionalización deportiva, diseñado en el país tras la segunda Guerra Mundial y destinado a crear campeones olímpicos en distintas disciplinas deportivas y a promover la práctica deportiva entre la población en general. El plan se mantuvo -con continuas mejoras- durante la existencia del país (Riordan, 1990; Morais \& Todd, 2013). En los EE.UU., en cambio, la planificación deportiva variaba en función de la ideología de la administración presidencial, por lo que hubo períodos de gran descoordinación deportiva a nivel federal. La falta de planificación durante el mandato del presidente Johnson (1963-1969), por ejemplo, repercutió considerablemente en los logros deportivos reflejados en el número de medallas olímpicas obtenidas- tanto durante su mandato como a lo largo de los años posteriores (Hunt, 2006). Otro elemento que podría influir en los resultados de las competiciones deportivas es el uso de las sustancias dopantes. Mientras que en la URSS y en la RDA el empleo de dichas sustancias por los deportistas se realizaba bajo la supervisión médica, los deportistas occidentales, debido a unas políticas antidopaje más restrictivas, se veían obligados a acudir al mercado negro, lo que ocasionaba mayores riesgos para su salud y podía provocar un descenso en su capacidad de rendimiento deportivo (Harries, 1985; Hunt et al., 2014).

\section{Conflicto de intereses}

Los autores declaran no tener ningún conflicto de intereses.

\section{Fuentes de financiación}

La presente investigación contó con la financiación de una Ayuda de Formación de Profesorado Universitario 2013 (FPU 2013) del Ministerio de Educación, Cultura y Deporte por parte de uno de los autores.

\section{Referencias}

Álvarez-Medina, J.; Manonelles-Marqueta, P.; Oliete-Blanco, E.; Murillo-Lorente, V. \& NuvialaNuviala, A. (2017). Validación de la escala de evaluación para la prevención del dopaje en escolares (CUPIAD). Retos, 32, 183-188.

Blázquez, D. \& Hernández, J. (1984). Clasificación o taxonomías deportivas. Barcelona: Monografía INEF.

Bolz, D. (2015). Sports Policy, the Press and the Origins of the Cold War in Occupied Germany, 1945 51. Sport In History, 35(2), 195-216.

Brundage,A. (1973). Prefacio. En Coubertin, P. de (autor). Ideario Olímpico (p. I). Madrid: Instituto Nacional de Educación Física.

Bucur, M. M.; Macovei, S. \& Margineantu, G. S. (2015). Sustainable development in the context of the olympic games. Ovidius University Annals, Series Physical Education \& SportScience, Movement \& Health.,15(2), 111-116.

Carreño-Ocaña, F. (2012). Citius, altius, fortius: la cara oculta de las medallas. Madrid: Atanor

Chinchilla-Marín, C. (2009). Los juegos olimpicos: la elección de la sede yotras cuestiones jurídicas. Cizur Menor (Navarra): Thomson Reuters-Civitas

Conlin, P. (1994). The cold war and canadian nationalism on ice: federal government involvementin international hockey during the 1960's. Canadian Journal Of History OfSport, 25 (2), 50-68.

Coubertin, P. de (1973). Ideario Olímpico. Madrid: Instituto Nacional de Educación Física. Harries, M. (1985). Deaths of athletes. British Medical Journal, 290, 656-657.

Holt, N. L. \& Neely, K. C. (2011). Positive youth development through sport: a review. Revista Iberoamericana de Psicología del Ejercicio yel Deporte, 6(2), 299-316.

Hunt, T. M. (2006). American Sport Policy and the Cultural Cold War: The Lyndon B. Johnson Presidential Years. Journal Of Sport History. 33 (3), 273-297.

Hunt, T. M.; Dimeo, P.; Hemme, F. \& Mueller,A. (2014). The Health Risks of Doping during the Cold War: AComparative Analysis of the Two Sides of the Iron Curtain. International Journal OfThe History OfSport, 31 (17), 2230-2244.

Kioussis, G. N. \& Hunt, T. M. (2015). Projection and reception: The American (ized) Other in East German Elite Sport, 1966-77. Journal of Sport History, 42(2), 161-178.

Laforge, F. (2012). Los Juegos Olimpicos: todas la olimpiadas de la era moderna. Barcelona: De Vecchi.

Martínez-Patiño, M. J.; Pena-Pérez, X. \& Mateos-Padorno, C. (2016). Percepción en adolescentes de los valores olímpicos asociados a la práctica del fútbol frente a otros deportes. Retos, 30, 226232.

McDougall, A. (2015). East Germany and the Europeanisation of football. Sport in History,35(4), 550

Meisinger, A. (2015). Der Olympiaboykott 1980. Eine vergleichende Analyse der Boykottdebatten in (West-)Europa. Zeitgeschichte, 42 (4), 233-249.

Morais, D. G., \& Todd, J. (2013). Lifting the Iron Curtain: Paul Anderson and the Cold War's First Sport Exchange. Iron Game History, 12 (2), 16-39.

Olivera-Betrán, J. (2012). Juegos Olímpicos Londres 2012: la olimpiada de las mujeres. Apunts. Educación Físicay Deportes, 109 (3), 7-10.

Pieper, L. P. (2014). Sex Testing and the Maintenance of Western Femininity in International Sport International Journal of the History of Sport, 31 (13), 1557-1576.

Platonov, V. N. (2001). Teoría general del entrenamiento deportivo olímpico. Barcelona: Paidotribo. Riordan, J. (1990). Playing to new rules: Soviet sport and perestroika. Soviet Studies, 42 (1), 133-145. Robles-Rey, E. \& Martínez-Núñez, L. V. (2013). Juegos Olímpicos: una propuesta didáctica e interdisciplinar. EmásF. Revista Digital de Educación Física, 4 (22), 72-91.

Rodríguez-Quijada, M. \& Molkova, S. (2016). Medallistas españoles en los Juegos Olímpicos de verano (1896-2012). EmásF. Revista Digital de Educación Física, 7(40), 79-86.

Soares, J. (2011). Guerra fria, gelo quente: hóquei no gelo internacional 1947-1980. Recorde: Revista De História Do Esporte, 4(1), 1-40.

Swift, J. (2008). Atlas histórico de la guerra fría. Madrid: Akal.

Thomas, D. (2007). Let the games begin: Sport, U.S. racerelations and Cold War politics. International Journal Of The History OfSport, 24(2), 157-171. 